Monatsschr Kinderheilkd 2009 157:13 DOI 10.1007/s00112-007-1653-z ๑) Springer Medizin Verlag 2009

Schriftleitung Monatsschrift Kinderheilkunde

\title{
Wissenschaftliche Gutachter für Monatsschrift Kinderheilkunde
}

\section{Dr. Wolfgang Eberl}

Städtisches Klinikum Braunschweig, Klinik für Kinder- und Jugendmedizin

PD Dr. Friedrich Ebinger

Universitätsklinikum Heidelberg, Zentrum für Kinder- und Jugendmedizin

Prof. Dr. Jochen Ehrich

Kliniken der MH Hannover

Dr. Joachim Eichhorn

Kinderklinik der Ruprecht-Karls-Universität, Heidelberg

Vestische Kinder- und Jugendklinik Datteln PD Dr. Susanne Bechtold-Dalla Pozza Kinderklinik und Kinderpoliklinik im Dr. von Haunerschen Kinderspital, München

Dr. Bernd Beedgen

Kinderklinik der Ruprecht-Karls-Universität, Heidelberg

Prof. Dr. Bernd Belohradsky Kinderklinik und Kinderpoliklinik im Dr. von Haunerschen Kinderspital, München

Dr. Marcus Benz

Kinderklinik und Kinderpoliklinik im Dr. von Haunerschen Kinderspital, München

Prof. Dr. Dietrich Berdel

Marien-Hospital gGmbH, Klinik für Kinder- und Jugendmedizin, Wesel

Dipl.-Psych. Dirk Bethe

Universitätsklinikum Heidelberg, Zentrum für Kinder- und Jugendmedizin

Dr. Christoph Bidlingmaier Kinderklinik und Kinderpoliklinik im Dr. von Haunerschen Kinderspital, München

Prof. Dr. Bernhard Bogerts Universitätsklinik für Psychiatrie, Psychotherapie und Psychosomatische Medizin, Magdeburg

PD Dr. Karl-Heinz Brisch Kinderpsychosomatik im Dr. von Haunerschen Kinderspital, München

PD Dr. Philipp Bufler

Kinderklinik und Kinderpoliklinik im Dr. von Haunerschen Kinderspital, München

Prof. Dr. Stefan Burdach Kinderklinik und Poliklinik des Klinikums Rechts der Isar derTU München

Dr. Martin Claßen

Klinikum Links der Weser, Zentrum für Kinder- und Jugendmedizin, Bremen

PD Dr. Robert Dalla Pozza Kinderklinik und Kinderpoliklinik im Dr. von Haunerschen Kinderspital, München

Prof. Dr. Hans-Georg Dietz Kinderchirurgische Klinik im Dr. von Haunerschen Kinderspital, München

Dr. Hildegard Doil

Bielefelder Institut für frühkindliche Entwicklung e.V., Bielefeld
PD Dr. Monika Führer

Kinderklinik und Kinderpoliklinik im Dr. von Haunerschen Kinderspital, München

Prof. Dr. Matthias Griese

Kinderklinik und Kinderpoliklinik im Dr. von Haunerschen Kinderspital, München

Dr. Jürgen Grulich-Henn

Kinderklinik der Ruprecht-Karls-Universität, Heidelberg

Dr. Dorothea Haas

Universitätsklinik für Kinder- und Jugendmedizin, Heidelberg

Prof. Dr. Helmut Hahn

Kinderklinik und Poliklinik des Klinikums Rechts der Isar derTU München

Prof. Dr. Erik Harms

Klinik und Poliklinik für Kinderheilkunde derWilhelms-Universität, Münster

Prof. Dr. Helge Uwe Hebestreit Universitätskinderklinik Würzburg

Prof. Dr. Florian Heinen

Kinderklinik und Kinderpoliklinik im Dr. von Haunerschen Kinderspital, München

Prof. Dr. Rolf D. Herzberg

Wuppertal

Dr. Stefan Hiedl

Frauenklinik, München

Prof. Dr. Hans-lko Huppertz Klinikum Bremen Mitte, Bremen

Dr. Daniela Kiepe

Kinderklinik der Ruprecht-Karls-Universität, Heidelberg

Prof. Dr. Christiane Kiese-Himmel Universitätsmedizin Göttingen

Prof. Dr. Berthold Koletzko

Kinderklinik und Kinderpoliklinik im Dr. von Haunerschen Kinderspital, München

Prof. Dr. Sibylle Koletzko

Kinderklinik und Kinderpoliklinik im Dr. von Haunerschen Kinderspital, München

Prof. Dr. Richard Kraemer Inselspital, Bern

Prof. Dr. Ingeborg Krägeloh-Mann Universitätsklinik für Kinderheilkunde und Jugendmedizin, Tübingen
Prof. Dr. Thomas Lang

Klinikum Starnberg, Klinik für Kinder- und Jugendmedizin, Starnberg

\section{Dr. Steffen Leiz}

Kinderklinik Dritter Orden, München

Dr. Henning Lenhartz

Wilhelmstift Katholisches Kinderkrankenhaus gGmbH, Hamburg

Prof. Dr. Ertan Mayatepek Universitätsklinikum Düsseldorf

Prof. Dr. Wilhelm Müller

Universitätsklinik für Kinder- und Jugendheilkunde, Graz

PD Dr. Elisabeth Mützel Institut für Rechtsmedizin, München

PD Dr. Mathias Nelle

Universitätskinderklinik, Inselspital, Bern

Prof. Dr. Heinrich Netz Klinikum der Universität München

Prof. Dr. Thomas Nicolai

Kinderklinik und Kinderpoliklinik im Dr. von Haunerschen Kinderspital, München

Prof. Dr. Charlotte Niemeyer Universitätsklinikum Freiburg

Dr. Jun Oh

Universitäts-Kinderklinik Düsseldorf

PD Dr. Jochen Peters

Kinderklinik Dritter Orden, München

Dr. Martina von Poblotzki

Frauenklinik, München

Prof. Dr. Christian Poets

Universitätsklinik für Kinderheilkunde und Jugendmedizin, Tübingen

Dr. Klemens Raile

Charité, Campus Virchow-Klinikum,

Klinik für Allgemeine Pädiatrie, Berlin

Prof. Dr. Ulrich Rappen

Klinik für Kinder und Jugendliche, Stadtklinik Baden-Baden

Prof. Dr. Wolfgang Rascher Universitätsklinikum Erlangen, Kinderund Jugendklinik

Prof. Dr. Reinhard Roos

Städtisches Krankenhaus München Harlaching, Klinik für Kinder und Jugendliche, München

PD Dr. Kevin Rostasy

Landeskrankenhaus Innsbruck, Universitätsklinik für Pädiatrie IV

Prim. Dr. Josef Rücker

Universitätsklinik für Kinder- und Jugendheilkunde, St. Johanns-Spital, Salzburg

PD Dr. Peter Ruef

Kinderklinik der Ruprecht-Karls-Universität, Heidelberg
PD Dr. Jens-Peter Schenk

Pädiatrische Radiologie des Universitätsklinikums Heidelberg, Heidelberg

\section{Prof. Dr. Hans Scherer}

Charité, Campus Benjamin Franklin, Zentrum für Audiologie und Phoniatrie, Augen- und HNO-Heilkunde, Berlin

Prof. Dr. Heinrich Schmidt

Kinderklinik und Kinderpoliklinik im Dr. von Haunerschen Kinderspital, München

Prof. Dr. Karl Schneider Kinderklinik und Kinderpoliklinik im Dr. von Haunerschen Kinderspital, München

Prof. Dr. G. Schott Urologische Universitätsklinik, Erlangen Prof. Dr. Hannsjörg Seyberth Landau

Dr. Arne Simon

Universitätsklinik, Zentrum für Kinderheilkunde, Bonn

Prof. Dr. Maximilian Stehr Kinderchirurgische Klinik im Dr. von Haunerschen Kinderspital, München

PD Dr. Daniel Steinbach Universitätskinderklinik Ulm

Prof. Dr. Gisela Szagun Universität Oldenburg, Institut für Psychologie

Dr. Torsten Uhlig

Klinikum Rosenheim, Klinik für Kinderund Jugendmedizin, Rosenheim

PD Dr. Maja von der Hagen Universitätsklinikum Carl Gustav Carus, Klinik und Poliklinik für Kinder- und Jugendmedizin, Dresden

Prof. Erika von Mutius

Kinderklinik und Kinderpoliklinik im Dr. von Haunerschen Kinderspital, München

Prof. Dr. Dietrich von Schweinitz Kinderchirurgische Klinik im Dr. von Haunerschen Kinderspital, München

Prof. Dr. Norbert Wagner Universitätsklinikum Aachen, Klinik für Kinder- und Jugendmedizin

Prof. Dr. Ulrich Wahn

Charité, Klinik für Pädiatrie mit Schw. Pneumologie und Immunologie, Berlin

Dr. Katharina Warncke

Kinderklinik und Poliklinik des Klinikums Rechts der Isar der TU München

PD Dr. Lutz Weber

Kinderklinik und Kinderpoliklinik im Dr. von Haunerschen Kinderspital, München 